\title{
Analyses on Potential Issues and Solutions of State-owned Power Enterprise Performance Management
}

\author{
Lei Zhao \\ ${ }^{1}$ HR Department, China Datang Corporation, Beijing 100033, China \\ Correspondence: Lei Zhao, No.1, Guangningbo Road, HR Department, China Datang Corporation, Beijing 100033, \\ China. Tel: 86-10-6658-6107. E-mail: zhaolei@china-cdt.com
}

Received: April 24, 2013

Accepted: May 8, $2013 \quad$ Online Published: July 2, 2013

doi:10.5430/ijba.v4n4p50

URL: http://dx.doi.org/10.5430/ijba.v4n4p50

\begin{abstract}
The Performance Management (PM) is one of the important approaches to improve the benefit achievements of power industry; however, the PM of electric power industry in China is still in the initial stage, under which it should be concerned as one of the most popular issues of electric power industry at present. This article analyzes the current circumstances of PM in electric power enterprise, and illustrates the existing issues and corresponding solutions, and also presents promotion and development prospects to our PM of Chinese state-owned power enterprise.
\end{abstract}

Keywords: state-owned power enterprise, performance analysis, performance management issue

\section{Current Situation Analyses of PM in Chinese State-owned Power Enterprise}

For the past few years, the Chinese electric power industry has developed very fast. In 2006, the general assets of the Chinese state-owned power enterprises are 4.3501 trillion RMB, and the sales income is 2.080811 trillion RMB, and the total profit is 144.58 billion RMB, which have been improved significantly than last year. At the meanwhile, our electric power industry is transforming from the situation of monopoly to competition, which means the competition between enterprises will also transform from obtaining the power resources to the whole ability of enterprise management. "The modern management theory and practice have helped us prove that the PM can not only realize the operation objective of enterprise in short term, but also play as one of the most important approaches to guarantee the core competitive ability for any enterprise." (1) Recently, the PM has been carried out in electric power industry, many enterprises have executed it promptly and positively, and a considerable number of enterprises have obtained periodical results. It experiences from the "pay attention to production safety" to "give consideration to the financial benefit and industry leading dual management", and then to the "legal person capital evaluation" and "state-owned power enterprise performance supervised by the SASAC (State-owned Assets Supervision and Administration Commission)" to the final "marketization PM" which are five kinds of model changes. Although the above five kinds of models contribute into the whole industry in different development periods, but the current position of overall power PM is still not ideal, and many issues exist in the PM of state-owned power enterprise.

\section{The Potential Issues of PM in State-owned Power Enterprise}

\subsection{Insufficient Instruction of Correct Concepts}

Human being is the primary resource of an enterprise, and the foundation of development. While the PM is the practical application based on the above of human resource strategy, via which we could distinguish the talents from the mediocre men, and also via implementing the performance appraisal system, will integrate the evaluation results of an employee with the salary treatment, position adjustment, training development, career planning, etc, to motivate employees to improve work performance, and finally to upgrade the overall performance of the enterprise and realize the mutual development of the company and employees. The PM, which implements the Enterprise Strategy, is an important management tool to promote the strategy execution capability of an enterprise, which has substantial difference from the traditional performance evaluation. It emphasizes on the management of execution results, at the meanwhile, it pays more attention to management and executive process. The PM requires the enterprise to complete their settled goals, and at the same time, it focuses on the balanced development of enterprises, and concerns to the continuous improvement of the enterprise performance. However, at present, although Chinese electric power industry enterprises have certain concerns on the importance of PM, from the current effects of PM 
execution, there is still quite a huge of concept deviation existed, indicates in:

1) The comprehension on the PM is limited to the performance assessment, without penetration into the daily management, project operation, which becomes formalistic and is unable to provide fully support in the sustainable development of the company. The current ongoing PM of state-owned power enterprise, in fact, is based on the administrative management mode. Such unified PM mode has not taken into account the characteristics of different business units, which leads to the uneven effects of PM. In addition, with delegating the right of operation management to lower levels, many state-owned power enterprises need to think about how to strengthen their "self management", which means, transform the custom of passive acceptance, and ensure all performance goals for different levels according to the enterprise development plans. The traditional performance appraisal usually focuses only on the assessment itself, which has not combined the performance assessment and the performance improvement, nor creates opportunities for PM to implement enterprise strategic goals.

2) The implementation of PM needs sorts of human beings, under the mode of division management, a comparative less number of administration people locates in each department, and most of them conduct multitasking, when the performance appraisal work is refining, a large number of statistical work will increase personnel's workload undoubtedly, which also needs further promotions on the business process. The streamlined work procedures for performance without enough participation and collaboration from subordinate units also lead to unreasonable objectives formulation, and the assessment of standards is more or less obscure, which caused the performance assessment to be formalistic.

3) Lack of long-term communication and daily management mechanism, which makes staffs feel passive to participate in, and have not connected their own career planning together, nor truly improve their performance; contemporarily, the performance appraisal is a sort of periodical work, as no performance information has been accumulated, such assessment is not objective, comprehensive, nor convincing, and correspondingly, the result is not easy to integrate with the motivation mechanism of salary adjustment, position adjustment, etc.

\subsection{Ignore the Construction of Ideology Environment}

The PM is a sort of systematic strategic management work, which needs a complete set of basic management system and regulations to support, so first of all is the transformation of thinking, via the top-down approach, from the top management to middle management and finally the ordinary employees, to promote step by step, and finally implement a comprehensive performance evaluation and the formation of the PM system. At the meanwhile, shall integrate the enterprise culture, development strategy, and personal career planning with PM together, making employees feel mutual development with the company together to create a win-win outcome. Let employees, the middles, and the tops hold hope for the future.

\subsection{Indeterminate Responsibilities between Management Team, Managers and Employees in Performance Management}

PM should be controlled and supervised by the top leader, as the core responsibility also should be bear by all top leaders together. But the reality is, it often becomes the "monodrama" for the HR Department, which significantly impacts the outcome effect. One of the root causes is: neither the leadership nor manager realizes that the PM is also part of their own jobs, but supposed that it should be conducted by HR Department, without the necessary responsibility realization, which directly caused performance deviations for all leadership. As different department leaders holds different management purposes, they always have different concerns for the same issue, which eventually leads to indeterminate responsibilities arise among the leadership members. The unclear responsibilities of PM execution in electric power industry indicate in:

1) As mentioned earlier, PM should be controlled and supervised by the top leader, so the effects of PM execution restrict to how much attention the leaders paid and promotion processed.

2) Generally, the present development of different departments are not balanced, and the management levels are also not equal, which makes it difficult to use the same set of measures to evaluate the personnel performance. Under such situation, the company should propose the instructive opinion to each division, and encourage the mature ones to further promote their evaluation rules and regulations to enhance the efficiency; for divisions who are processing under the initial stage of development, as they have less personnel and more extension pressure, with less human resource investment, can simplify the operation procedure based on instruction suggestions. 
3) Usually, the HR department undertakes the PM work, which should be conducted by the other departments; correspondingly the other departments begin to believe that PM is none of their business and is titled to be executed by HR specialists, which reduces the working efficiency of the whole HR department.

4) For all ordinary employees, as they do not know about the PM work very well, and no specific realizations stimulated between the PM and themselves, which depresses their participation enthusiasm. Under such situation, the PM work can't effectively extend to the most grass-roots staff of a company, reducing the outcome effects of PM implementation.

\subsection{Lack of Objective Measure Standard, Pay Too Much Attention on the Past Performance, and Backward Matched System}

PM is a systemic project, and it cannot be separated from other management systems to work independently, especially relating to the enterprise culture, strategic management and organization management, etc, which are close with the enterprise important strategies. And correspondingly, the management system, evaluation system, rewards and punishment system will generate great influence on the PM work. If those systems are not perfect enough, nor completely associated with the PM system, will affect the operation of the PM system and operation effect directly. And whether all rules and regulations including performance appraisals of the PM system standard are appropriate will also contribute to the final decision. In the implementation process of PM, defects exist, which indicate in:

1) Some evaluation objectives of PM system are too abstract and cannot represent the real performance result. For example, if there is a high assessment percentage in "Loyalty", "Attitude", and "Compliance" those qualitative indexes then performance results are very hard to tell, and ultimately the performance evaluation result also may not be objective. The one without specific evaluation measures will be even worse than nothing. As the evaluation results based on the impression, feel, even the private relationship, are lack of objectivity, and may even affect the leadership and the harmonious between the leader and the subordinates. So specific assessment standards are crucial to the existence value of PM system.

2) The inner spirit and cognition of PM decide whether the enterprise has the capability of foresight, and whether they can make use of PM system to effectively enhance staff work efficiency. The existing performance evaluation system of state-owned power enterprise usually pay more attention to the past, less to the future; focus on the results instead of the process. So each assessment is based on the past performances, and do not pay attention to the ongoing innovation and improvement work, which reduce the passion and motivation of the development of enterprises.

3) Other supporting management systems are not complete. For instance, the system of salary management, employee career management, staff promotion management, training management, are not completed nor combined closely to the PM system, making which loses its proper driving force, and will affect the final operation of the PM system.

\section{How to Establish a Perfect PM System for a State-owned Power Enterprise}

From the above analyses, we can tell that the state-owned power enterprise must focus on the following in order to establish a perfect PM system

\subsection{The Cognition on the Concept of PM Should Be Integrated}

As the electric power industry and the performance management work start quite late, and also restricted by the traditional management concept and management mechanism, we should have a transformed understanding on PM cognition via the following learning processes:

1) Invite external experts: the PM work is a systematic management project, and the cognition should be established internally from top to bottom initially, so the external experts should familiar with the management of the electric power system, especially the PM work. Provide PM training to internal top management leadership and mid-level managers, so that they can apply PM skills in daily management work to improve the PM level of the whole company.

2) Popularization publicity: after integrating the cognition of PM between top management and core managers of mid-level, realizing the importance of PM and applying the PM work into the company duly management, popularize the summarization of actual operation issues of management process, examination system, and evaluation indexes to all staff pervasively, makes the PM interiorized to employees and their daily work.

3) Organize regular internal training: manage the performance assessment of a company as a strategy management systematic project, refrain from formalism, and select leadership from functional departments 
to train related staff on rules and regulations of performance assessment management, technical process, and propagandize important PM evaluation work.

4) Sum up rewards and punishments regularly: pay rewards to outstanding departments who have good consequences of PM work, and promote excellent PM idea and the successful experience; also conduct certain punishment measures on those departments who carried out PM slowly and ineffectively, so that establish an effective competition mechanism to maximize the outcome of PM evaluation.

\subsection{To Strengthen the Management of PM Process}

\subsubsection{Select the Right PM Method}

At present, main mature PM methods are: key performance indicators method, target management, and the balanced scorecard method, etc. The choice of PM methods had better integrate with the operational characteristics and development stage. For functional department of an electric power company, we can use the target management. On determining the main participant of PM, it is necessary to practice diversification, realize the competent leadership (or higher level departments), associated department, associated post multi-aspect appraisal. The state-owned power enterprise is a typical state-owned enterprise, the assessment might easily be affected by private relationships, so the multi-aspect of PM evaluation is helpful to solve such kind of issues.

\subsubsection{Confirm the Target of PM}

Here in fact concerns two level issues, the so-called performance index options and the determination of its performance. For the performance indicators, firstly we must respect the personalized management, which according to different types of department or position, choose the appropriate index system. Secondly, the choice of the PM index of the enterprise had better consider both short-term and long-term goals. The determination of the performance target for quantitative indicators can consider the overall target of different layers based on layer decomposition, and qualitative indexes should be decided based on the communications from top to bottom of leadership.

\subsubsection{Implementation Informatization Management}

Informatization management of PM has become an irresistible trend, drive and promote the enterprise performance management level, and increase the enterprise value multifaceted. Specifically, innovation and value of PM information system indicate in the following aspects: is the strategy management tools for the innovation of information; promote enterprise PM system construction; make performance evaluation more transparent, fair and impartial; make the operation of PM more sustainable, and ensure long-term operation; not only can solve the creative problems, but also the bottleneck and core of enterprise management. Via an electronic PM system, we can achieve the unification of company goals and performance; make the storage and exchange employee data more convenient; can approach to promote to remind information management process and reduce the routine operation time of PM, in order to realize the optimization of the utilization of human resources; can improve the capability of performance tracking and reporting, and provide decision support and guidance function online.

3.2.4 PM Should Combine with the Enterprise Culture, Employing Mechanism and Other Aspects of the State-owned Power Enterprise Management

We must solve the concept problem first, driven by the model of senior management team, form the compliance system habit among management team and general staff, make employee progress behavior become a cultural atmosphere, which in turn improve the healthy development of our enterprise. Secondly, provide system safeguard is also an important aspect. A complete performance management system should include the following elements: assessment goals and objectives, evaluation principles, object scope, organization and leadership, execution relationship, cycle of evaluation, evaluation content, evaluation method and procedures, steps of process, requirements of evaluation result, application of appraisal result and feedback, matters needs attention, channel of information storage, examination system and explain the authority of the content. Another important aspect is accurate key performance index, we should make enterprise and employees are voluntary to perform the key performance indicators, according to the enterprise actual situation.

\subsection{The Prospects on PM of State-owned Power Enterprise}

Where is the promotion opportunity for Chinese state-owned power enterprise PM development? And what is the future trend of PM development? For Chinese enterprises, PM has just started, at the same time; it is still a long way to go for the development of the state-owned power enterprise performance management. To improve the PM of the electric power industry, we might commence from the following aspects: first, revise the ongoing PM system, such 
as index extraction, weight reset, application models, etc., to make the PM system fit with the development strategy, and closer to the employees' expectations, transforming the performance appraisal from the position of passive to positive. Secondly, should pay more attention to the process management and control, keep supervision and control before or during the procession other than after the completion, and set up a perfect control and communication system. Then, as a minor cycle management system, the PM should be combined with the knowledge management, accumulate all produced documents and knowledge, to implement the value of the relay based on the analyses. Finally, in the practical application of PM, try to reduce the management cost as much as possible and provide a more convenient operation environment to employees.

In conclusion, the future trend of PM in power industry is focus on the initial stage of performance conduction, to help employees achieve the desired outcome via the index supervision, performance communication, knowledge management, and the cultivation of ability. And perform portal conductions in the actual applications, and create a personalized and humanized operating environment to employees.

\section{References}

Fang, Shaohua, \& Hu, Yingying. (2007). Consulting of performance management. Mechanical Industry Press.

Fang, Zhenbang. (2007). Strategic performance management. Beijing: China Renmin Press.

Li, Jian. (2005). Human resource management practices necessary manual. Chinese Yanshi Press.

Wang, Zhiyu. (2003). Performance rubik's cube: performance management operation manual. China Social Sciences Press. 